\title{
Energy-Efficiency Joint Cooperative Spectrum Sensing and Power Allocation Scheme for Green Cognitive Radio Network: A Soft Decision Fusion Approach
}

\author{
Nouhoum Satarou Abdoul Galeb Yari ${ }^{1,2,}$, Mbembo Loundou Varus ${ }^{1}$, Dong Doan Van ${ }^{1,2}$ \\ ${ }^{1}$ School of Information Engineering, Wuhan University of Technology, Wuhan, China \\ ${ }^{2}$ Key Lab Broadband Wireless Communication and Sensor Networks, Ministry of Education, Wuhan University of Technology, Wuhan, \\ China
}

\author{
Email address: \\ yasmigab@yahoo.fr (N. S. A. G. Yari), loundouvarus@yahoo.fr (M. L. Varus), dongdoan@yahoo.fr (D. D. Van) \\ ${ }^{*}$ Corresponding author
}

\section{To cite this article:}

Nouhoum Satarou Abdoul Galeb Yari, Mbembo Loundou Varus, Dong Doan Van. Energy-Efficiency Joint Cooperative Spectrum Sensing and Power Allocation Scheme for Green Cognitive Radio Network: A Soft Decision Fusion Approach. American Journal of Networks and Communications. Vol. 7, No. 2, 2018, pp. 6-16. doi: 10.11648/j.ajnc.20180702.11

Received: May 22, 2018; Accepted: July 1, 2018; Published: August 2, 2018

\begin{abstract}
This paper focus on a jointly spectrum sensing parameter and energy efficiency (EE) optimization problem in OFDMA CRN system for enabling Green Communication. In this perspective, we firstly propose an algorithm to choose less spatially-correlated cognitive users to reduce the shadowing effect in wireless network. Furthermore, based on Lagrangian duality theorem with the aid of parametric transformation, the algorithm called an Iterative Dinkelbach Scheme (IDS) is proposed to optimize both transmission power allocation and sensing duration of the cognitive users (Cus) for maximizing Energy Efficiency under the constraints of overall outage of cognitive network, interference to the PU, maximum transmission power and minimum data rate requirement. Numerical result proves the effectiveness of our proposed algorithm. Compared with existing schemes, our proposed scheme outperforms in enhancing the EE with the same parameters.
\end{abstract}

Keywords: Cognitive Radio, Green Communication, Energy Efficiency, IDS Algorithm, Dinkelbach Method, Lagrangian Duality, Less Spatially-Correlated

\section{Introduction}

One of the most important challenges in dealing with the ever-increasing service demands in future wireless communication is the shortage of radio resources. Cognitive Radio Network (CRN) which provides opportunistic access of Primary users's band (licensed spectrum) to the secondary users, is considered as a promising new paradigm with great potential of improving the spectrum radio utilization. In order to efficiently combat the effects of attenuation, shadowing and the hidden layer problem in a pratical wireless network, the Cooperative Spectrum Sensing (CSS) was introduced in CRNs [1]. In this system, the fusion center (FC) combines the local detection results from secondary users following two strategies: Hard decision fusion (HDF) [2, 3] and Soft Decision fusion (SDF) $[4,5]$. According to literature review, the performance of SDF-based CSS schemes is better than
HDF- based CSS schemes [4, 5]. This mainly motivated us to formulate our resource allocation model in a SDF-based detection scheme. The design of the energy-efficient green CRN depends on the system throughput and the power consumed by the sensing devices. Meanwhile, the detection time must be chosen to maintain a compromise between the accuracy of spectrum detection and system throughput [6]. Optimal power allocation is also needed to limit the unnecessary growth of transmit power that can introduce severe interference to the primary user [7]. Specifically, in wireless network, the transmission power degrades with the distance raised to the power of the path-loss factor. The reduced weak signal forces the SUs to falsely decide that there is no PU in the desired band and to transmit their data. The more Secondary users there are, the more energy is consumed. Thus, the exclusion of these shadowed SUs and the inclusion of adequate SUs (less spatially-correlated) can 
improve the probability of detection with less energy consumption.

\subsection{Related Works}

most papers in the literature have addressed power allocation algorithms with limited interference to the Primary User in order to preserve energy efficiency. In [10], $\mathrm{Wu}$ and al. Formulate a jointly optimizing sensing time and power allocation problem under the constraints of sensing parameters and interferences to the PU [8]. The authors in [9] proposed a power allocation algorithm by taking detection error into consideration for OFDMA-based cooperative networks. In [10], an energy-efficient model was suggested for multiple amplify-and-forward relays in single sourcedestination CR System. Here, the EE maximization problem is formulated as a minimization of the total energy consumption under the constraints of false alarm probability, the detection probability, the Cognitive Users data rate and the minimum interference threshold. A new iterative algorithm based on the parametric transformation method has been proposed in [11] for the green CR under the interference constraint at PU and has been extended for the green cooperative based on the decoded-forward relay (DF) in [12] under both interference and SUs outage constraints. To determine the optimal power allocation strategies on different fading channels, Kang et al. considered the PU's channel state information and maximized the outage capacity at cognitive network under the constraints of the maximum transmission power from the SUs and outage probability of PU [13]. In [14], the EE variation over the detection time was formulated as a concave optimization, so golden section search method was used to find the optimal detection time for the maximum EE. Most papers are based on HDF-based CSS and don't take into account PU activity during the data transmission period when formulating the interference model. This paper focus on maximizing EE with optimal detection time and power allocation with limited interference to Primary User and outage of SUs.

\subsection{Contributions}

The main contributions of this work are threefold and summarized as follow:

(a) at first we implement an interference-aware model taking in consideration the exponential transition probabilities of PU during the data transmission time slot.

(b) Secondly, based on Hungarian method, we propose an algorithm to select less spatially-correlated SUs

(c) Thirdly, we formulate this joint optimization problem using two approaches:

i) $\mathrm{We}$ propose an Iterative Dinkelbach Scheme (IDS) algorithm under the constraints of differents parameters associated with EE maximization

ii) In IDS algorithm, the sensing time is determined using golden section search method at each iteration for maximum throughput and the sub-problem associated with the power allocation is resolved by utilizing Karush-Kahn-Tucker
(KKT) condition on Lagrangian dual problem.

Finally, a detailed analysis of the performance of the proposed scheme is presented with the simulations results.

Table 1. List of symbols.

\begin{tabular}{ll}
\hline Symbol & description \\
\hline $\mathrm{T}_{0}$ & Data transmission duration \\
$\mathrm{P}\left(\mathrm{H}_{1}\right)$ & PU presence Probability \\
$\mathrm{P}\left(\mathrm{H}_{0}\right)$ & PU absence Probability \\
$\Gamma_{t h}$ & Outage threshold \\
$\mathrm{K}$ & Total number of SUs \\
$\mathrm{X}_{\mathrm{i}, \mathrm{j}}$ & Element of correlation matrix X \\
$\mathrm{R}_{\mathrm{th}}$ & Minimum achievable data rate \\
$P_{\text {ogei }}$ & Outage of $\mathrm{i}^{\text {th }}$ Cognitive users \\
$\alpha$ and $\beta$ & Busy and idle rate \\
$\mathrm{M}$ & Selected cognitive users \\
$u$ & Dinkelbach parameter \\
$\tau_{\mathrm{s}}$ & Sensing time \\
$\mathrm{N}_{0}$ & Power of noise \\
$\mathrm{P}_{\mathrm{Pr}}$ & Interference power received from PU \\
\hline
\end{tabular}

the table above (Table 1) gives information about the different symbols that are used for this work and their respective meanings

\section{System Model and Problem Formulation}

This section is devoted to the CSS system model based on SDF, and the interference model with the aim to evaluate the total possible interference during the data transmission time slot.

\subsection{System Modeling}

We consider a CRN consists of $\mathrm{K}$ SUs which are uniformly distributed around the centrally located PU from which only $\mathrm{M}$ less spatial -correlated Cognitive Users are assumed to be selected. They jointly perform spectrum sensing on the entire licensed band. The licensed is then divided into $\mathrm{M}$ number of sub-bands so as to allow each SU to transmit its data separetly in one of the sub-bands to its corresponding cognitive destination (CD). Let a CR system consists of Q number of frames each of length $\mathrm{T}$. Each frame consists of sensing slot of duration $\tau_{s}$, reporting slot of duration $\tau_{r}=\sum_{i=1}^{M} r_{i}$ and one data transmission slot of duration $T_{0}=T-\tau_{r}-\tau_{s}$. We assume that $\tau_{r}$ is very less as compared to $\tau_{s}$ and $T_{0}$ hence is neglected in further part of the evaluation. All M Cus each with single energy detector (ED) receive PU's signal and send their local sensing results to the FC. If $\mathrm{z}$ $(\mathrm{t})$ is the signal from the PU, then the received signal $x_{i}(k)$ at $i^{\text {th }} \mathrm{SU}$ in $k^{\text {th }}$ instant is formulated as two binary hypotheses tests.

$$
x_{i}(k)=\left\{\begin{array}{cc}
\phi_{i}(k), & \mathrm{H}_{0} \\
z(k) h_{p s i}(k)+\phi_{i}(k), & \mathrm{H}_{1}
\end{array} \quad i=[1,2, \ldots \ldots . M]\right.
$$

where $\phi_{i}(k)$ is the additive noise with zero mean and variance 
$\sigma_{\phi_{i}^{2}}^{2}$

The PU signal is assumed to be independent and identically distributed (i.i.d.) of zero mean and variance $\sigma_{z^{2}}$. The propagation channels between the PU and the SUs follow the path-loss and log-normal distributions which is given by $h_{p s i}(k) \sim\left(d_{i}\right)^{-w} e^{x}$. Here, $d_{i}$ is the distance between the PU and $i^{\text {th }} \mathrm{SU}, \mathrm{w}$ is the path-loss exponent and $x$ is the Gaussian random variable with mean and variance $\sigma^{2}$, where $\sigma=0.1 \ln (10) \sigma_{d B}$ and $\sigma_{d B}$ is the log-normal distribution and its range is $4 \leq \sigma_{d B} \leq 12$. The two hypotheses $\mathrm{H}_{0}$ and $\mathrm{H}_{1}$ represent the absence and presence of the PU, respectively. Each SU measures the received power over $N$ samples during the sensing interval and makes the local decision as follows

$$
G_{i}=\frac{1}{N} \sum_{k=0}^{N-1}\left|x_{i}(k)\right|^{2}
$$

where $N=\tau_{S} f_{S}$ and $f_{S}$ is the sampling frequency. According to the central limit theorem, for large value of $N$ the local test statistic is approximately represented by the Gaussian distribution which is given by [5]

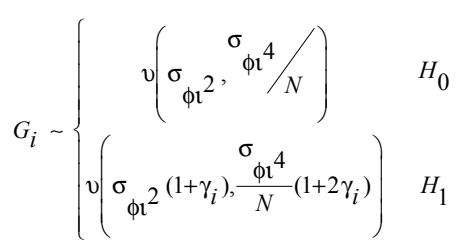

where $\gamma i$ is the received SNR at the $i^{\text {th }}$ SU and is given by $\gamma_{i}=\frac{\sigma_{s_{2}}\left|h_{p s i}\right|^{2}}{\left|\sigma_{\phi 1}\right|^{2}}$. If $\lambda$ denotes the threshold, then the probability of detection and false alarm of the $i^{\text {th }} \mathrm{CU}$ are given by

$$
\begin{gathered}
P_{\operatorname{det}_{i}}\left(\tau_{s}\right)=P_{r}\left(G_{i} \succ \lambda \mid \mathrm{H}_{1}\right)=Q\left(\frac{\lambda-\sigma_{\phi \mathrm{l}}{ }^{2}\left(1+\gamma_{i}\right)}{\sigma_{\phi \mathrm{l}} 2 \sqrt{1+2 \gamma_{i}}} \sqrt{\tau_{s} f_{s}}\right) \\
P_{f a_{i}}\left(\tau_{s}\right)=P_{r}\left(G_{i} \geq \lambda \mid \mathrm{H}_{0}\right)=Q\left(\frac{\lambda-\sigma_{\phi \mathrm{l}^{2}}}{\sigma_{\phi \mathrm{l}^{2}}} \sqrt{\tau_{s} f_{s}}\right)
\end{gathered}
$$

respectively. FC linearly combines the local test statistics with the weight coefficients of SUs to make the global decision statistic $G_{G l e}$ which is expressed as [5]

$$
G_{G l e}=\sum_{i=1}^{M} w_{i} G_{i}
$$

where $w_{i}$ is the weight coefficient assigned to $i^{\text {th }} \mathrm{SU}$. An external constraint is added to the weight vector $w=\left[w_{1}, w_{2}, \cdots w_{M}\right]^{T}$ such that $w_{2}^{2}=1$. The weight vector can be optimized by using MRC [6], EGC [6] and MDC [5]. Here, we consider $w_{i}=\frac{\gamma_{i}}{\sqrt{\sum_{i=1}^{M} \gamma_{i}^{2}}}$ because of its suitability in low SNR condition [6].

Then, the final decision is evaluated by comparing $G_{\text {Gle }}$ with the global threshold $\lambda_{g}$ and is expressed as [5]

$$
P_{\operatorname{det}_{i}}\left(\tau_{S}\right)=Q\left(\frac{\lambda_{g}-\sum_{i=1}^{M} \sigma_{\phi \mathrm{l}}{ }^{2}\left(1+\gamma_{i}\right) W_{i}}{\sqrt{\sum_{i=1}^{M} \sigma_{\phi \mathrm{l}}{ }^{4}\left(1+2 \gamma_{i}\right) W_{i}^{2}}} \sqrt{\tau_{S} f_{S}}\right)
$$

and

$$
P_{f a_{i}}\left(\tau_{s}\right)=Q\left(\frac{\lambda_{g}-\sum_{i=1}^{M} \sigma_{\phi \mathrm{t}}{ }^{2}{ }_{i}}{\sqrt{\sum_{i=1}^{M} \sigma_{\phi \mathrm{l}}{ }^{4} W_{i}^{2}}} \sqrt{\tau_{s} f_{s}}\right)
$$

From (7), the corresponding false alarm probability for the given detection probability can be determined as

$$
P_{f a_{i}}\left(\tau_{s}\right)=Q\left[a+b \sqrt{\tau_{s} f_{s}}\right]
$$

where $a$ and $b$ are given by $a=\frac{Q^{-1}\left(P_{\operatorname{det}_{i}}\right) \sqrt{\sum_{i=1}^{M} \sigma_{\phi t^{4}}\left(1+2 \gamma_{i}\right) W_{i}^{2}}}{\sqrt{\sum_{i=1}^{M} \sigma_{\phi 1} W_{i}^{2}}}$ and $b=\frac{\sum_{i=1}^{M} \sigma_{\phi 1^{2}} W_{i} \gamma_{i}}{\sum_{i=1}^{M} \sigma_{\phi 1} W_{i}^{2}}$

\subsection{Interference-Aware Modeling}

The activity of the PU is modeled as an exponentially distributed ON-OFF process which alternates between ON and OFF state. If $\alpha$ and $\beta$ represent the busy and idle rates, then the probability that the channel is being occupied by the PU is $P\left(H_{1}\right)=\frac{\alpha}{\alpha+\beta}$, and the probability that PU is absent is $P\left(H_{0}\right)=\frac{\beta}{\alpha+\beta}$. During the data transmission time slot, interference to the PU may be expected in two possible scenarios. In the first scenario, SUs correctly detect the absence of the PU during sensing period but the PU makes a transition in the data transmission period. In this case, the possibility of PU being present during $T_{0}$ is given by [17]

$$
P_{01}\left(T_{0}\right)=\frac{\beta}{\alpha+\beta}\left(T_{0}+\frac{e^{\left[-(\alpha+\beta) T_{0}\right]}}{\alpha+\beta}\right)
$$

Hence, the interference for this OFF state is calculated as $I_{O F F}=\left(1-P_{f a_{i}}\right) P_{01}\left(T_{0}\right)$. In the second scenario, SUs fail to identify the weak PU signal and start transmitting their data. So, the possibility of PU presence during $T_{0}$ is expressed as [17] 


$$
P_{11}\left(T_{0}\right)=T_{0}-\frac{\alpha}{\alpha+\beta}\left(T_{0}+\frac{e^{\left[-(\alpha+\beta) T_{0}\right]}-1}{\alpha+\beta}\right)
$$

So, the interference in this busy state is given by ${ }^{I} O N=P_{m d} P_{11}\left(T_{0}\right)$, where $P_{m d}$ denotes the missed detection probability. The average interference introduced to the PU is defined as the ratio of total interference occurs during $T_{0}$ with the persistence of PU signal during the entire frame.

$$
I_{\text {Total }}=\frac{I_{O N}+I_{O F}}{P\left(H_{1}\right) T}
$$

\section{Optimization Problem Formulation}

\subsection{The Problem Formulation for Throughput Maximization}

Based on the FC final decision, there are two possible scenarios when the SUs intend to transmit their data to the CDs. In the first scenario, the PU is correctly detected to be absent in the desired band. So, the channel capacity achieved at $i^{\text {th }} \mathrm{CU}$ is $c_{0 i}=\log _{2}\left(1+\left|h_{s d i}\right|^{2} \frac{p_{t i}}{N_{0}}\right)$. Here, $h_{s d i}$ is the Rayleighdistributed sub-channel gain between the $i^{\text {th }} \mathrm{SU}$ and its corresponding $\mathrm{CD}$, and is calculated as $h_{s d i} \sim N\left(0, \frac{1}{d_{i}^{z}}\right) \cdot p_{t i}$ represents the transmission power from the $i^{t h} \mathrm{CU}$ and $N_{0}$ denotes the noise power. In the second scenario, the PU is active on the licensed band, but the SUs unable to identify the PU signal and start transmitting their data. The capacity achieved in this case is expressed as $c_{1 i}=\log _{2}\left(1+\frac{\left|h_{s d i}\right|^{2} p_{t i}}{\left|h_{p d i}\right|^{2}+P_{p}+N_{0}}\right)$, where $P_{\operatorname{Pr}}$ is the interference power due to presence of PU received at the secondary network and $h_{p d i}$ is the Rayleigh-distributed sub-channel gain between the PU and $i^{\text {th }} \mathrm{CD}$, and is calculated as $h_{p d i} \sim N\left(0, \frac{1}{d_{i}^{z}}\right)$. Hence, $P_{\operatorname{Pr}}$ is given by:

$$
P_{\operatorname{Pr}}=\frac{P\left(H_{1}\right) P_{m d}}{P\left(H_{0}\right)} \sigma_{s}^{2}
$$

where $P\left(\bar{H}_{0}\right)$ denotes the total probability of event $\bar{H}_{0}$ which is given by $P\left(H_{0}\right)=\left(1-P_{f a}\right)+P\left(H_{1}\right) P_{m d}$. Under these two scenarios, the average throughput achieved at the secondary CR network is [8]

$$
R_{s y s}\left(\tau_{s}, P_{t}\right)=\sum_{i=1}^{M} R_{1 i}\left(\tau_{s}, P_{t}\right)+\sum_{i=1}^{M} R_{2 i}\left(\tau_{s}, P_{t}\right) \approx \sum_{i=1}^{M} R_{1 i}\left(\tau_{s}, P_{t}\right)
$$

$$
\begin{aligned}
& \sum_{i=1}^{M} R_{1 i}\left(\tau_{s}, P_{t}\right)=\left(1-P_{f a}\right) P\left(H_{0}\right) C_{0 i} \frac{T_{0}}{T} \\
& \sum_{i=1}^{M} R_{2 i}\left(\tau_{s}, P_{t}\right)=P\left(H_{1}\right) P_{m d} C_{1 i} \frac{T_{0}}{T}
\end{aligned}
$$

From (14), it is clear that longer sensing time reduces the data transmission period. If $R_{t h}$ denotes the minimum achievable data rate of $i^{\text {th }} \mathrm{SU}$, then the minimum transmission power from each $\mathrm{SU}$ is expressed as

$$
P_{t i} \geq \frac{\left(2^{R_{t h}}-1\right) N_{0}}{\left|h_{s d i}\right|^{2}}
$$

Then, we analyze the overall outage performance to examine the effect of interference on the system throughput. An outage occurs when the channel capacity of the SU falls below the threshold $R_{t h}$. So, the outage at $i^{\text {th }} \mathrm{SU}$ is given by

$$
P_{\text {ogei }}=\frac{T_{0}}{T}\left[P\left(H_{0}\right)\left(1-P_{f a}\right)\left(1-e^{\frac{-\gamma_{t h}}{\sigma_{s d i}^{2}}}\right)+P\left(H_{1}\right) P_{m d}(\underbrace{2}_{p d i^{2} \gamma_{P} \gamma_{t h}+\sigma_{s d i}^{2}} e^{\frac{-\gamma_{t h}}{\sigma_{s d i}^{2}}})\right]
$$

where $\gamma_{P}=P_{\operatorname{Pr}} / N_{0}$ and $\gamma_{t h}=\frac{\left(2^{R_{t h}}-1\right) N_{0}}{P_{t i}}$. Then the outage probability constraint of $i^{\text {th }} \mathrm{CU}$ is expressed as

$$
P_{\text {ogei }} \leq \Gamma_{\text {th }}
$$

where $\Gamma_{t h}$ is the outage threshold. The main objective of this paper is to maximize EE by jointly optimizing the sensing time and power allocation $P_{t}=\left[P_{t 1}, P_{t 2}, \ldots P_{t M}\right]$. The first suboptimization problem is the throughput maximization which is formulated as

$$
\begin{gathered}
\text { maximize: } R_{s y s}\left(\tau_{s}, P_{t}\right) \\
\text { subject to: } P_{d} \geq P_{d} \\
0 \leq \tau_{s} \leq T \\
P_{t i} \geq \frac{\left(2^{R_{t h}}-1\right) N_{0}}{\left|h_{s d i}\right|^{2}} \\
P_{\text {ogei }} \leq \Gamma_{t h}
\end{gathered}
$$

For any value of $P_{t}, R_{s y s}\left(\tau_{s}\right)$ is a unimodal function of $\tau_{s}$, it increases monotonically from $0 \leq \tau_{s} \prec \tau_{s}$ and decreases $\tau_{s}{ } \leq \tau_{s} \prec T$. So, there exists a unique value of sensing time $\tau_{s}$ at which $R_{\text {sys }}\left(\tau_{s}\right)$ is maximum.

Theorem 1. $R_{s y s}\left(\tau_{s}\right)$ is a concave function of $\tau_{s}$, where $\tau_{s}$ * 
gives the maximum average throughput. it is proved that $R_{s y s}\left(\tau_{s}\right)$ is a unimodal function in the range $0 \leq \tau_{S} \prec T$. Hence, golden section search method is employed to get optimal sensing time for maximum throughput [18].

\subsection{Energy Efficiency Maximization Problem}

The second optimization problem is to maximize EE. In the CRNs, essentially the power is consumed during the spectrum sensing and data transmission, and obviously the sensing power is less than the data transmission power. So the total power consumption in the secondary network (CR system) can be derived as: (neglecting the circuit power consumption)

$$
P_{T}\left(\tau_{s}, P_{t}\right)=\tau_{s} M P_{S}+T_{0} P\left(H_{0}\right) \sum_{i=1}^{M} P_{t i}
$$

where $P_{s}$ is the sensing power and assumed to be same for all the SUs. Then, EE (bit/s/joule) of the CRNs is defined as the ratio of the average throughput (14) and the total power consumption by the secondary network (25) which is expressed as

$$
E E_{\text {eff }}\left(\tau_{s}, P_{t}\right)=\frac{R_{\text {sys }}\left(\tau_{s}, P_{t}\right)}{P_{T}\left(\tau_{s}, P_{t}\right)}
$$

The EE maximization problem is formulated under the following constraints

$$
\begin{gathered}
\underset{\tau_{s}, P}{\text { Maximize }} \frac{R_{\text {sys }}\left(\tau_{s}, P_{t}\right)}{P_{T}\left(\tau_{s}, P_{t}\right)} \\
\text { s.t. }: P_{t \max }-\sum_{i=1}^{M} P_{t i} \geq 0 \\
: I_{t h}-I_{\text {total }} \sum_{i=1}^{M}\left|h_{s p i}\right|^{2} P_{t i} \geq 0 \\
: \Gamma_{t h}-P_{\text {ogei }} \geq 0 \\
: C_{0 i} \geq R_{t h} \\
: P_{t i} \geq \frac{\left(2{ }_{t h}-1\right) N_{0}}{\left|h_{s d i}\right|^{2}} \\
: 0 \leq \tau_{s} \leq T
\end{gathered}
$$

where $P_{t}=\left[P_{t 1}, P_{t 2}, \ldots P_{t M}\right] . P_{t \max }$ is the maximum allowable transmission power from the SUs and $I_{t h}$ represents the interference power threshold. Represents the interference power threshold. The total interference power below $I_{t h}$ will not affect the legitimate PU. Here, $h_{s p i}$ is the sub-channel gain between the $i^{\text {th }} \mathrm{SU}$ and the PU.

\section{Solution Approach}

In this section, we develop a less spatially-correlated SUs selection algorithm to select M SUs out of the total K SUs. Then, the joint optimization of sensing and transmission power allocation is obtained through our proposed IDS algorithm.

\subsection{SUs Selection Method}

Shadowing produces spatially weak and correlated signals on the secondary networks. It has already been proved that less number of SUs in a large area is more effective than more numbers of SUs in a dense area [19]. These uncorrelated SUs can be selected adaptively [20] or from its normalized covariance matrix of received signal [21]. Let $K$ be the total number of SUs present in a CRN, then the element in the correlation matrix $X$ is expressed as [22]

$$
X_{i, j}=e^{-\varepsilon d_{i j}}
$$

where, $d_{i j}$ represents the distance between the $i^{t h}$ and the $j^{\text {th }}$ CU. $\varepsilon$ is an environmental constant. $\varepsilon \approx 0.1209 / \mathrm{m}$ and $\varepsilon \approx 0$. $002 / \mathrm{m}$ in the urban environment and in the suburban environment, respectively. To avoid the effect of the spatial correlation, we propose a SUs selection algorithm which is based on Hungarian method [23]. The steps describing the algorithm are given as follows.

\subsection{Iterative Dinkelbach Scheme (IDS) for Resource Allocation}

We adopt the nonlinear fractional or parametric programming to solve the objective function (23). As per parametric programming, if $p(\chi)$ and $q(\chi)$ are continuous and real valued functions and $\chi \in S$, where $S$ is a subset, then, $\max _{\chi \in S}\left\{\frac{p(\chi)}{q(\chi)}\right\}$ can be transformed to $\max _{\chi \in S}\{p(\chi)-u q(\chi)\}$. This transformation relies on the following theorem.

Theorem 2. If $\mathrm{S}$ is the feasible set then there exists a optimal objective function such that if and only if

$$
\begin{gathered}
u^{*}=\frac{p\left(\chi^{*}\right)}{q\left(\chi^{*}\right)}=\max _{\chi \in S}\left\{\frac{p(\chi)}{q(\chi)}\right\} \\
\max _{\chi \in S}\left\{p(\chi)-u^{*} q(\chi)\right\}=p\left(\chi^{*}\right)-u^{*} q\left(\chi^{*}\right)=0
\end{gathered}
$$

As per this theorem, our optimization problem is formulated as $\quad u^{*}=\frac{R_{s y s}\left(\tau_{s}{ }^{*}, P_{t}{ }^{*}\right)}{P_{T}\left(\tau_{s}{ }^{*}, P_{t}{ }^{*}\right)}=\max \left\{\frac{R_{s y s}\left(\tau_{s}, P_{t}\right)}{P_{T}\left(\tau_{s}, P_{t}\right)}\right\} \quad$ and $\max \left\{R_{\text {sys }}\left(\tau_{s}, P_{t}\right)-u^{*} P_{T}\left(\tau_{s}, P_{t}\right)\right\}=R_{\text {sys }}\left(\tau_{s}{ }^{*}, P_{t}{ }^{*}\right)-u^{*} P_{T}\left(\tau_{s}{ }^{*}, P_{t}{ }^{*}\right)=0$

Here, $\tau_{s}{ }^{*}$ and $P_{t}{ }^{*}$ represent the optimal sensing time and optimal power allocation, respectively. Our proposed IDS algorithm presents the steps for joint optimization of sensing time and power allocation with suitable selection of SUs from Algorithm 1. According to Algorithm 2, at each 
iteration iter, $P_{t}($ iter $)$ is calculated from the sub-problems associated with the convex optimization which is given in the next sub-section. Then, for the given transmission power, sensing time is found out for maximum (20) by using golden section search method. Algorithm 2 is terminated when $u($ iter $)$ converges.

\subsection{Sub-problem for Optimal Power Allocation}

In $(26), R_{\text {sys }}\left(\tau_{s}, P_{t}\right)$ is a concave and $P_{T}\left(\tau_{s}, P_{t}\right)$ is an affine function of SUs transmission power. Also the constraints given in $(28-33)$ are either linear or convex. Hence, for the given $\tau_{s}$, the optimal power allocation problem $\left\{R_{\text {sys }}\left(\tau_{s}, P_{t}\right)-u^{*}(\right.$ iter $\left.) P_{T}\left(\tau_{s}, P_{t}\right)\right\}$ can be formulated using the convex theory subject to the (29), (30) and (32) constraints. Then, this sub-optimization problem is solved by Lagrangian duality theorem which is expressed as

$$
\begin{aligned}
& L(\delta, \rho, v)=C \sum_{i=1}^{M} \log _{2}\left(1+\frac{\left|h_{s p i}\right|^{2} P_{t i}}{N_{0}}\right)-u(i t e r)\left(\tau_{s}^{M P_{s}+T_{0} P\left(H_{0}\right)}-\sum_{i=1}^{M} P_{t i}\right)+\delta\left(P_{t \max }-\sum_{i=1}^{M} P_{t i}\right)+ \\
& \rho\left(I_{t h}-I_{\text {total }} \sum_{i=1}^{M}\left|h_{\text {spi }}\right|^{2} P_{t i}\right)+v_{i}\left(\log _{2}\left(1+\psi_{i} P_{t i}\right)-R_{t h}\right) \\
& \text { s.t. : } P_{t \max }-\sum_{i=1}^{M} P_{t i} \geq 0 \\
& : I_{\text {th }}-I_{\text {total }} \sum_{i=1}^{M}\left|h_{s p i}\right|^{2} P_{t i} \geq 0 \\
& \log _{2}\left(1+\psi_{i} P_{t i}\right)-R_{t h} \geq 0
\end{aligned}
$$

where $\delta, \rho$ and $v=\left[v_{1}, v_{2}, \ldots . v_{M}\right]$ are the non-negative Lagrangian multipliers, $\psi_{i}=\frac{\left|h_{s d i}\right|^{2}}{2 N_{0}}$ and $C^{\prime}=\left(1-P_{f a_{i}}\right) P\left(H_{0}\right) \frac{T_{0}}{T}$. In each iteration iter of Algorithm 2, the Lagrangian dual problem is solved such that $\min _{\delta, \rho, v} \max _{\tau_{s}, P_{t}} L(\delta, \rho, v)$.

Then by applying KKT condition to this dual problem we have [25]

$$
\begin{gathered}
\frac{C^{\prime} \psi_{i}}{\left(1+\psi_{i} P_{t i}\right)(\ln 2)}-u(i \text { ter }) T_{0} P\left(H_{0}\right)-\delta-\rho I_{\text {total }} \sum_{i=1}^{M}\left|h_{s p i}\right|^{2}+v_{i} C_{0 i}^{\prime}=0 \\
\delta\left(P_{t \max }-\sum_{i=1}^{M} P_{t i}\right)=0 \\
\rho\left(I_{\text {th }}-I_{\text {total }} \sum_{i=1}^{M}\left|h_{s p i}\right|^{2} P_{t i}\right)=0 \\
v_{i}\left(\log _{2}\left(1+\psi_{i} P_{t i}\right)-R_{t h}\right)=0
\end{gathered}
$$

where $C_{0 i}^{\prime}=\frac{\psi_{i}}{\left(1+\psi_{i} P_{t i}\right)(\ln 2)}$. It is clearly observed from (42), (43) and (44) that each condition generates two cases; either the dual variable is zero or the corresponding constraint is zero. So, we have summarized the possibilities into four cases, and accordingly calculate $P_{t}($ iter $)$ for the current iteration iter of Algorithm 2

Case 1: $\delta=\rho=0$ and $v_{i}=0$ for all $i \in M$. So, (28) reduces to

$$
\begin{gathered}
\frac{C^{\prime} \psi_{i}}{\left(1+\psi_{i} P_{t i}\right)(\ln 2)}-u(\text { iter }) T_{0} P\left(H_{0}\right)=0 \\
\text { s.t. }: P_{t \max }-\sum_{i=1}^{M} P_{t i} \geq 0 \\
: I_{\text {th }}-I_{\text {total }} \sum_{i=1}^{M}\left|h_{s p i}\right|^{2} P_{t i} \geq 0 \\
\log _{2}\left(1+\psi_{i} P_{t i}\right)-R_{t h} \geq 0
\end{gathered}
$$

The power solution of $i^{\text {th }} \mathrm{SU}$ from (41) is obtained by water filling solution and is expressed as

$$
P_{t i}^{1}=\left[\frac{C^{\prime}}{u(i t e r) T_{0} P\left(H_{0}\right) \ln 2}-\frac{1}{\psi_{i}}\right]^{+}
$$

Where $[.]^{+}=\max [0,$.$] . Then, substituting the value of (49)$ in (46), (47) and (48), following three conditions (50), (51) and (52) are evaluated, respectively which are as follows

$$
\begin{gathered}
\frac{C^{\prime}}{u(\text { iter }) T_{0} P\left(\overline{H_{0}}\right) \ln 2} \leq \frac{P_{t \max }}{M}+\frac{1}{M} \sum_{i=1}^{M} \frac{1}{\psi_{i}} \\
\frac{C^{\prime}}{u(i \text { iter }) T_{0} P\left(\overline{H_{0}}\right) \ln 2} \leq \frac{I^{*}+\sum_{i=1}^{M} \frac{\left|h_{s p i}\right|^{2}}{\psi_{i}}}{\sum_{i=1}^{M}\left|h_{s p i}\right|^{2}} \\
\frac{C^{\prime}}{u\left({ }^{2}\right.} \geq \frac{\left({ }^{R} t h\right)}{\psi_{i}}
\end{gathered}
$$

Where $I^{*}=\frac{I_{\text {th }}}{I_{\text {total }}}$. If (50), (51) and (52) are satisfied, then (49) is the power allocation solution

Case-2: $\delta \neq 0, \rho=0$ and $v_{i}=0$ for all $i \in M$. Similar to the Case-1, (41) reduces to

$$
\begin{gathered}
\frac{C^{\prime} \psi_{i}}{\left(1+\psi_{i} P_{t i}\right)(\ln 2)}-u(i \text { iter }) T_{0} P\left(H_{0}\right)-\delta=0 \\
\text { s.t. } \sum_{i=1}^{M} P_{t i}=P_{t \max } \\
: I_{\text {th }}-I_{\text {total }} \sum_{i=1}^{M}\left|h_{\text {spi }}\right|^{2} P_{t i} \geq 0 \\
\log _{2}\left(1+\psi_{i} P_{t i}\right)-R_{t h} \geq 0
\end{gathered}
$$


In this case, the optimal solution for power allocation is obtained as

$$
P_{t i}^{2}=\left[\frac{C^{\prime}}{\delta+u(i t e r) T_{0} P\left(H_{0}\right) \ln 2}-\frac{1}{\psi_{i}}\right]^{+}
$$

Therefore, (51) and (52) are satisfied, and (50) is reduced to equality constraint which is given by

$$
\frac{C^{\prime}}{u(\text { iter }) T_{0} P\left(\overline{H_{0}}\right) \ln 2}=\frac{P_{t \max }}{M}+\frac{1}{M} \sum_{i=1}^{M} \frac{1}{\psi_{i}}
$$

The water filling solution in (57) consists of the dual variable $\delta$ in the denominator which is updated by subgradient method as per the following expression [25]

$$
\delta(i t+1)=\left[\delta(i t)-Y(i t)\left(P_{t \max }-\sum_{i=1}^{M} P_{t i}\right)\right]^{+}
$$

where $Y$ ( it ) is non-negative step size for the current iteration it and is set at $1 / \sqrt{i t}$. So, $Y(i t)$ decreases with the number of iteration and $\eta$ converges to its optimal value when $Y($ it $)$ is sufficiently small.

Case-3: $\delta=0, \rho \neq 0$ and $v_{i}=0$ for all $i \in M$. Then the KKT condition given in (37) is reduced to:

$$
\begin{gathered}
\frac{C^{\prime} \psi_{i}}{\left(1+\psi_{i} P_{t i}\right)(\ln 2)}-u(i t e r) T_{0} P\left(H_{0}\right)-\rho I_{\text {total }} \sum_{i=1}^{M}\left|h_{s p i}\right|^{2}=0 \\
\text { s.t. }: I_{\text {th }}=I_{\text {total }} \sum_{i=1}^{M}\left|h_{s p i}\right|^{2} P_{t i} \\
: P_{t \max }-\sum_{i=1}^{M} P_{t i} \geq 0 \\
\log _{2}\left(1+\psi_{i} P_{t i}\right)-R_{t h} \geq 0
\end{gathered}
$$

After solving ( 60 ), the power allocation solution is given by

$$
P_{t i}^{3}=\left[\frac{C^{\prime}}{\left(u(\text { iter }) T_{0} P\left(\overline{H_{0}}\right)+\rho I_{\text {total }} \sum_{i=1}^{M}\left|h_{s p i}\right|^{2}\right) \ln 2}-\frac{1}{\psi_{i}}\right]^{+}
$$

With the conditions ( 50 ) and ( 52 ) are satisfied, and ( 51 ) is satisfied with equality

$$
\frac{C^{\prime}}{u(i t e r) T_{0} P\left(\overline{H_{0}}\right) \ln 2}=\frac{I^{*}+\sum_{i=1}^{M} \frac{\left|h_{s p i}\right|^{2}}{\psi_{i}}}{\sum_{i=1}^{M}\left|h_{s p i}\right|^{2}}
$$

Similar to Case-2, the solution (64) consists of the dual variable $\rho$ in the denominator which is updated by subgradient method as per the following expression

$$
\rho(i t+1)=\left[\rho(i t)-Y(i t)\left(I_{t h}-I_{\text {total }} \sum_{i=1}^{M}\left|h_{s p i}\right|^{2} P_{t i}\right)\right]^{+}
$$

So, $\rho$ converges to the optimal value when $Y$ (it) is sufficiently small.

Case 4: $v_{i} \neq 0$ for all $i \in M, \delta=0$ and $\rho=0$. For this case, (41) reduces to

$$
\begin{gathered}
\frac{C^{\prime} \psi_{i}}{\left(1+\psi_{i} P_{t i}\right)(\ln 2)}-u(i t e r) T_{0} P\left(H_{0}\right)+v_{i} C_{0 i}^{\prime}=0 \\
\text { s.t. } \log _{2}\left(1+\psi_{i} P_{t i}\right)=R_{t h} \\
: \frac{C^{\prime} \psi_{i}}{\left(1+\psi_{i} P_{t i}\right)(\ln 2)}-u(i t e r) T_{0} P\left(H_{0}\right)=0 \\
P_{t \max }-\sum_{i=1}^{M} P_{t i} \geq 0
\end{gathered}
$$

After Solving (67), we have the water filling solution

$$
P_{t i}^{4}=\left[\frac{C^{\prime}}{\left(u(i t e r) T_{0} P\left(H_{0}\right)-v_{i} C_{0 i}^{\prime}\right) \ln 2}-\frac{1}{\psi_{i}}\right]^{+}
$$

Therefore, $P_{t i}^{4}$ in (71) consists of dual variable $v_{i}$ in the denominator. But, there is no common water level for this solution. However, we have approached this solution in a clever way. In this case, the optimal solution occurs on the plane in (68) which is equivalent to

$$
P_{t i}^{4}=\left[\frac{C^{\prime}}{u(i t e r) T_{0} P\left(\overline{H_{0}}\right) \ln 2}-\frac{1}{\psi_{i}}\right]^{+} \text {or } P_{t i}^{4}=\left[\frac{2 R_{t h}}{\psi_{i}}-\frac{1}{\psi_{i}}\right]^{+}
$$

We have checked all the inequalities conditions given in (50), (51) and (52). Also, we have evaluated the exact power allocations for these four cases. Then, we incorporate these four cases into a single Algorithm 3 to decide transmission power $P_{t}$ for Algorithm 2. For convenience, we consider that

$$
\begin{aligned}
& P_{1}=\frac{C^{\prime}}{u(i t e r) T_{0} P\left(\overline{H_{0}}\right) \ln 2}, P_{2}=\frac{P_{t \max }}{M}+\frac{1}{M} \sum_{i=1}^{M} \frac{1}{\psi_{i}}, P_{3}=\frac{I^{*}+\sum_{i=1}^{M} \frac{\left.h_{s p i}\right|_{i}}{\sum_{i=1}^{M}\left|h_{s p i}\right|^{2}},}{P_{4}=\frac{\left(2^{R} t h\right)}{\psi_{i}} .}
\end{aligned}
$$

Algorithm 3 explains the exact power allocation to $i^{\text {th }} \mathrm{SU}$. Similarly, $P_{t}$ is evaluated for all $M$ Sus 


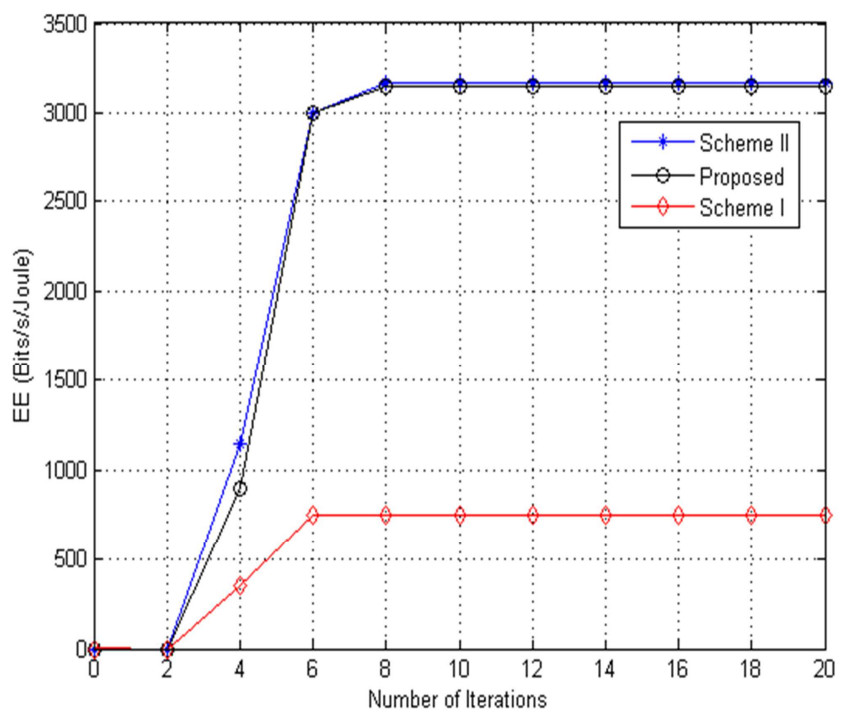

Figure 1. Convergence comparison of our proposed scheme with Scheme I and Scheme II.

\section{Simulation Results}

This section is devoted to numerical results in order to evaluate the performance of our proposed algorithm taking in account of different systems key parameters. It's considered a CRN where K SUs coexist with centrally located PU in a circular area of radius $1 \mathrm{~km}$. $\mathrm{K}$ is assumed to be 20 , among them only 6 SUs ( ie $M=6$ ) are supposed to be less spatialcorrelated. The CDs are distributed randomly around the PU over the same circular area of radius $1.5 \mathrm{~km}$. We consider $\sigma_{\mathrm{s}}^{2}$ $=1$. Table 2 summarizes some common parameters used in our simulation.

Table 2. Simulation Parameters.

\begin{tabular}{ll}
\hline Simulation parameters & value \\
\hline Noise power $N 0$ & $10^{-3}$ Watt \\
Sensing power $P$ s & 0.02 Watt \\
Path-loss coefficient $z$ & 4 \\
$\alpha$ & 0.5 \\
$\beta$ & 0.5 \\
Frame duration $T$ & $0.01 \mathrm{~s}$ \\
Target detection probability ${ }^{-} P d$ & 0.9 \\
$\gamma_{i} \forall \in M$ & $-20 \mathrm{~dB}$ \\
Sampling frequency & $6 \mathrm{MHZ}$ \\
Minimum tolerable value $\xi$ & $10^{-5}$ \\
$\sigma_{d B}$ & 4 \\
\hline
\end{tabular}

Table 3. SUs selection algorithm.

Input: $K$, the total number of Sus
$X=\left[\begin{array}{cccc}x_{1,1} & x_{1,2} & \ldots & x_{1, k} \\ x_{2,1} & x_{2,2} & \ddots & x_{2, k} \\ \vdots & & \cdots & \vdots \\ x_{k, 1} & x_{k, 2} & \cdots & x_{k, k}\end{array}\right] X$ is defined as a correlation matrix of size $(K \times K)$
1 find out the minimum value of each row and substract it from that row.
If at least one zero appear at each row, then
Follow step 3 else follow step 2
2: likewise, find out the minimum value of
each column and substract it from that column
3: cover zeros with minimum number of lines

drawn horizontally (row wise)

and vertically (column wise). If

the number of lines is equal to $\mathrm{K}$, then go to step7

4: Find out the minimum value among the uncovered elements

and add it to the covered elements. If any of the elements is covered twice,

then add minimum value to that element twice.

5: substract the minimum value of the matrix from each element

6: mask the zero elements by drawing horizontal and vertical lines.

If the number of lines is not equal to the number of rows, go to step 4

7: zero elements in the matrix represent the minimum spatial-correlation

value made by $i_{t h} \mathrm{SU}$ with the $j_{t h} \mathrm{SU}$ or vice versa

8: choose the rows or columns containing maximum number of zeros.

These selected rows represent the less spatially- correlated Sus M

\section{Table 4. IDS algorithm}

Input: $u=$ Dinkelbach parameter, $\xi=$ Accepted tolerance value, iter $=$

Current iteration,

$1:$ Initialize: $u \rightarrow 0, \xi \rightarrow 10^{-5}$, iter $\rightarrow 0$

$P_{t}$ satisfying the constraints (23a) $-(23 f)$ and find out $\tau_{s}$ for maximum

throughput using

2: Repeat

3: Using $\tau_{s}$ evaluate power $P_{t}($ iter $)$ from Algorithm 3 as given follows.

$P_{t}=\arg \max \left\{R_{s y s}\left(\tau_{s}, P_{t}\right)-u(\right.$ iter $\left.) P_{T}\left(\tau_{s}, P_{t}\right)\right\}$

4: for the giving $P_{t}($ iter $)$ determine the corresponding sensing time

5: iter $=$ iter +1

6: Calculate $u($ iter $)$ as per $\left\{\frac{R_{s y s}\left(\tau_{s}, P_{t}\right)}{P_{T}\left(\tau_{s}, P_{t}\right)}\right\}$

7: Return the optimal sensing time $\tau_{s}{ }^{*}$ and transmission power $P_{t}{ }^{*}$

8: Until $\mid u($ iter +1$)-u($ iter $) \mid \leq \xi$

Figure 1 reprents the comparison of our proposed scheme with two others schemes. In scheme I, the sensing time and transmission power allocation of SUs are jointly optimize through the iterative process discussed in $[13,14]$. In scheme II, the method of EE maximization problem formulation and the method of solving the power allocation through Lagrangian duality theorem are adopted from [10]. But the joint optimization of both sensing time and power allocation is done by using our Algorithm 2 instead of using two separate algorithms as in [10]. In scheme II, the exact power allocation for step 3 in Algorithm 2 is derived through KKT condition by solving (27). The other simulation parameters of this Figure 1 are $\mathrm{M}=6$, outage threshold $\Gamma_{t h}=0.05, R_{t h}=2$ $\mathrm{bits} / \mathrm{sec} / \mathrm{hz}$ and $I_{t h}=-20 \mathrm{dBW}$. It can be deduced that our proposed scheme give better performances comparing with the scheme I and scheme II

Table 5. Power allocation algorithm.

$\left(\frac{C^{\prime}}{u(i t e r) T_{0} P\left(\overline{H_{0}}\right) \ln 2}\right)$ is evaluated for $u($ iter $)$ obtained from Algorithm 2.
Input: $\mathrm{P}_{1}, \mathrm{P}_{2}, \mathrm{P}_{3}, \mathrm{P} 4$
1: if $\left(\left(P_{1} \prec P_{2}\right) \& \&\left(P_{1} \prec P_{3}\right) \& \&\left(P_{1} \succ P_{4}\right) \|\left(P_{1}=P_{2}\right) \& \&\left(P_{1}=P_{3}\right)\right)$ then $P_{t i}=P_{t i}^{1}$
2: elseif $\left(P_{1}=P_{2}\right) \& \&\left(P_{1} \prec P_{3}\right) \& \&\left(P_{1} \succ P_{4}\right) \quad P_{t i}=P_{t i}^{2}$
3: elseif $\left(P_{1} \prec P_{2}\right) \& \&\left(P_{1}=P_{3}\right) \& \&\left(P_{1} \succ P_{4}\right) \quad P_{t i}=P_{t i}^{3}$



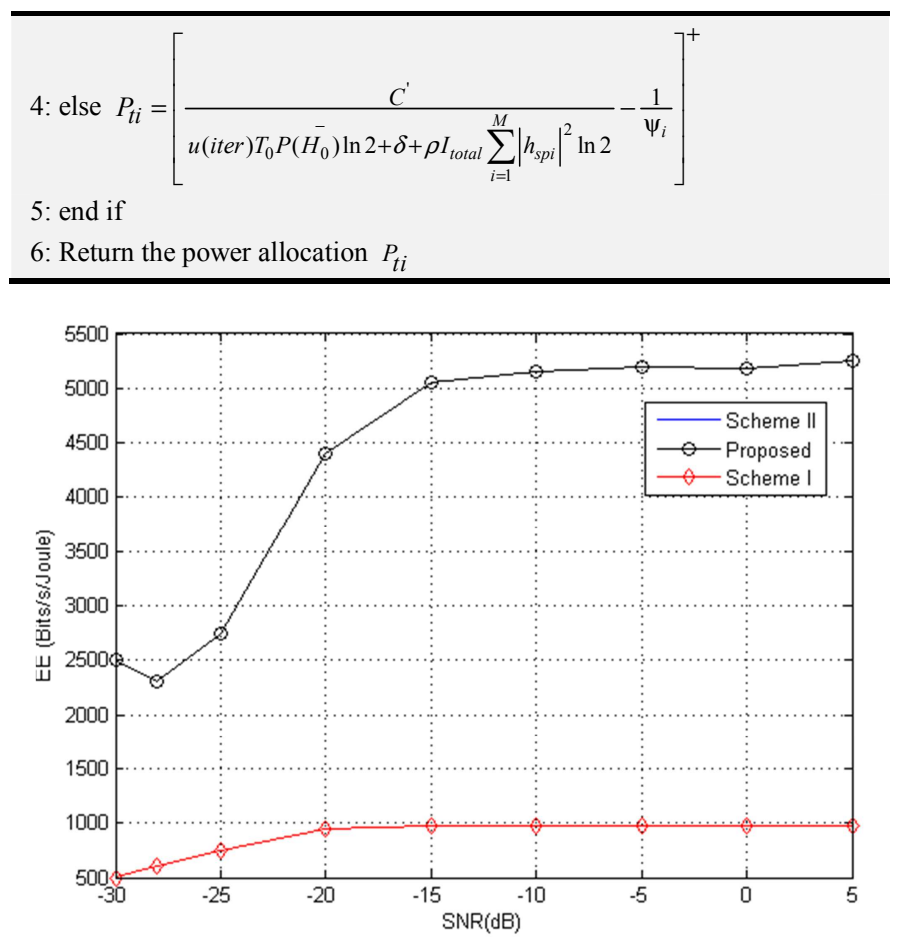

Figure 2. Performance comparison of our proposed scheme with Scheme I and Scheme II.

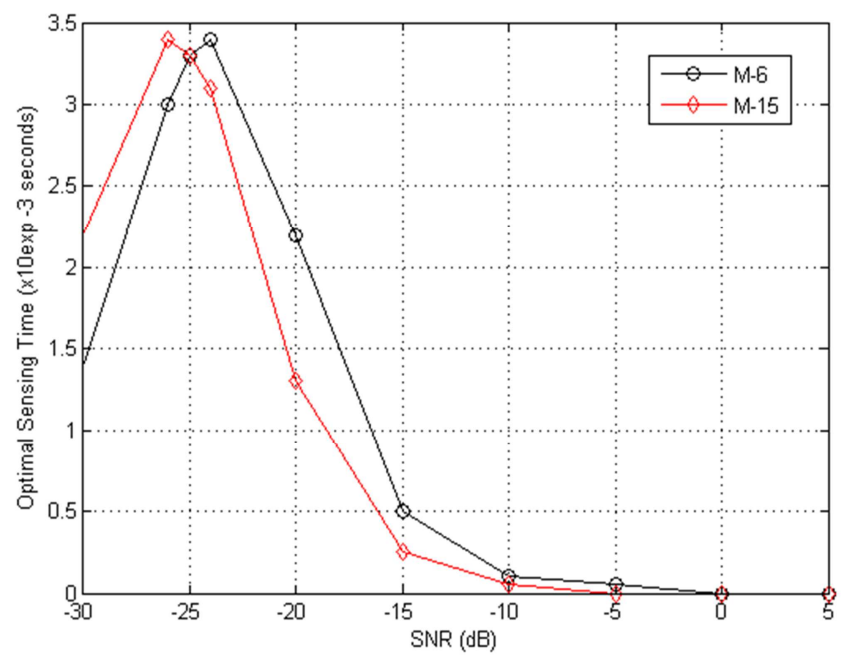

Figure 3. Optimum sensing time versus $S N R$ for $M=6$ and $M=15$.

Figure 2 shows the variation of maximum EE over differents SNR values for $I_{t h}=-20 \mathrm{dBW}$ of scheme I, scheme II and proposed scheme. It is clearly observed that our method performs almost the same as scheme II but gives better results than scheme I. In terms of performance our method gives significant improvement in EE with the same simulation parameters. Therefore, both Algorithms 2 and 3 jointly provides better performance with considerably less complexity. The simulation parameters remains the same as the parameters used to obtains Figure 1.
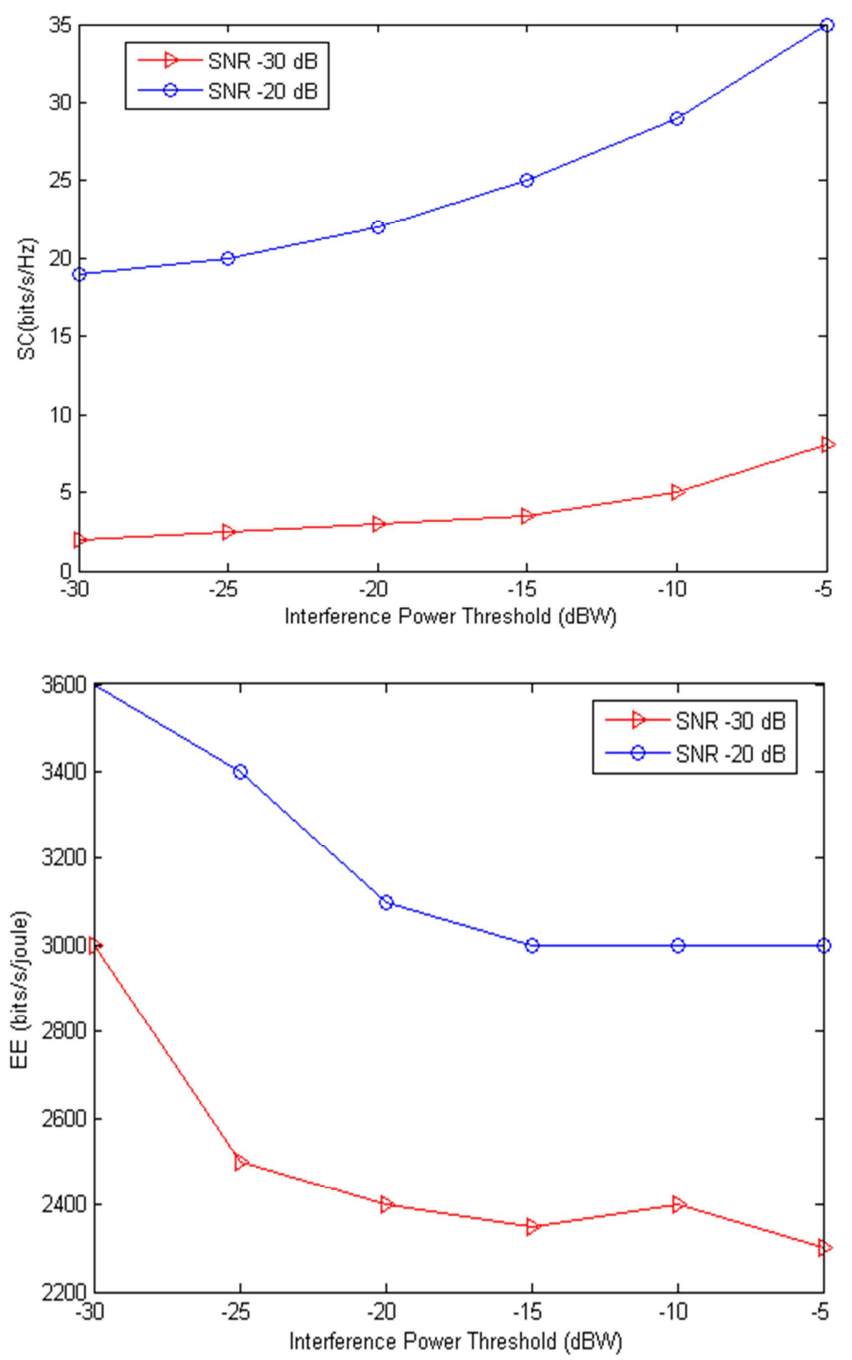

Figure 4. Maximum EE and maximum $S C$ versus outage threshold $I_{\text {th }}$ for $S N R=-20 d B W$ and $-30 d B W$.

Figure 3 illustrates the optimal spectrum detection time for $\mathrm{M}=6$ (less spatial-correlated cooperative SUs) and $\mathrm{M}=15$ (randomly selected SU). It is obvious that the higher the value of the SNR is, the less the SUs require sensing time. In addition, for a value of SNR $=-24 \mathrm{~dB}$, nearly $3.4 \mathrm{~ms}$ over 10 $\mathrm{ms}$ are used for spectrum detection, which thus occupied a large part of the total frame leaving a very short duration for data transmission. Therefore, the capacity of the system (SC) decreases at low SNR. The simulation parameters are the same as in Figure 1

Figure 4 illustrates the effect of interference power threshold $I_{t h}$ on the maximum EE and maximum SC for SNR $=-20 \mathrm{~dB}$ and $-30 \mathrm{~dB}$. System parameters for simulation are $M=6, \Gamma_{t h}=0.09$ and $R_{t h}=2 \mathrm{bits} / \mathrm{s} / \mathrm{Hz}$. It is noticed that with increase in $I_{t h}$, EE decreases and SC increases. Although it enhances the SC, Increasing in $I_{t h}$ is not always an advantage for EE maximization. Because with increase in $I_{t h}$ the total transmission power boundary increases as per (23b), which ultimately maximizes SC. 

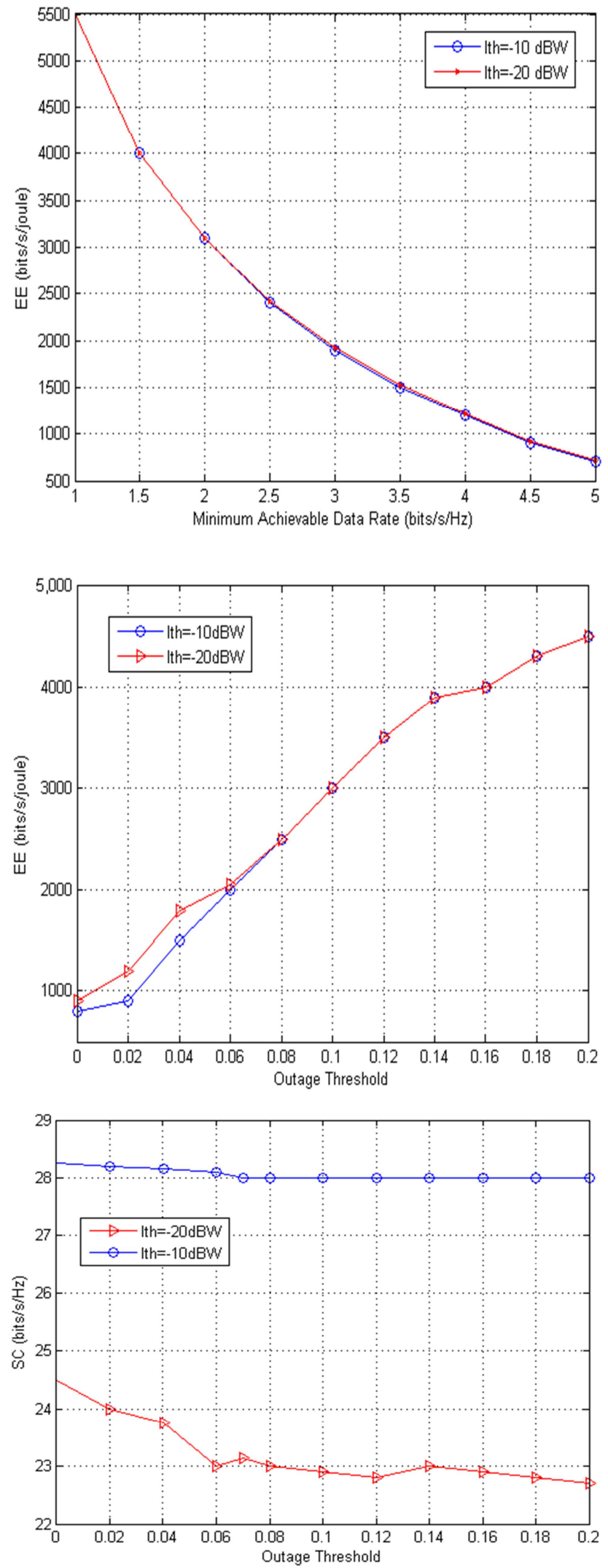

Figure 5. Maximum EE and maximum $S C$ versus outage threshold $\Gamma_{\text {th }}$ for $I_{\text {th }}=-20 \mathrm{dBW}$ and $I_{\text {th }}=-10 \mathrm{dBW}$.

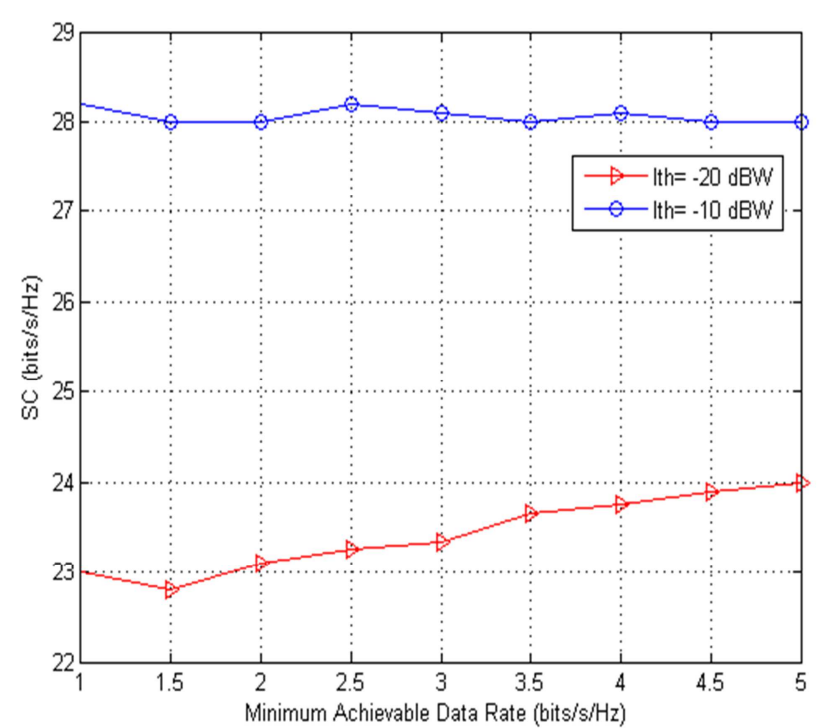

Figure 6. Maximum EE and $S C$ versus minimum achievable data rate $R_{\text {th }}$ for $I_{\text {th }}=-20 \mathrm{dBW}$ and $I_{\text {th }}=-10 \mathrm{dBW}$.

Figure 5 illustrates the effect of outage threshold $\Gamma_{t h}$ on the maximum $\mathrm{EE}$ and maximum $\mathrm{SC}$ for $\mathrm{M}=6$ and $R_{t h}=2$ bits $/ \mathrm{s} / \mathrm{Hz}$. We observe that $\mathrm{EE}$ increases with increase in outage threshold but SC does not show very significant changes. Hence, more outage threshold leads to a better framework for energy-efficient model keeping the system throughput almost unaffected.

Figure 6 presents the effect of number of cooperative Cus on maximum $\mathrm{EE}$, maximum $\mathrm{SC}$ and total transmission power for $I_{t h}=-20 \mathrm{dBW}$ and $=I_{t h}-10 \mathrm{dBW}$. It is obvious that EE decreases with increase in the number of Cus but SC increases as total transmission power increases. More SUs lead to more power consumption. However, the rate of decrease in EE does not change significantly after a certain number of Cus though the transmission power increases. Hence, an energy-efficient model can be designed by selecting suitable number of SUs to avoid unnecessary growth of power consumption. For this figure, SUs are chosen randomly from K SUs. The other simulations parameters are the same as the parameters previously used in Figure 1.

\section{Conclusion and Future Research}

Environmental, financial, and QoE challenges have motivated research in the area of energy efficient green communication system. In this paper, the problems of sensing time optimization in the SDF-based CSS and transmission power allocation in CRN is investigated with the aim of maximizing the energy efficiency thereby reducing the cost and sensing duration. Our approach guaranteed the EE maximization throughout the proposed IDS algorithm which optimizes jointly the sensing time and power allocation with less-spatially-correlated SUs. The selection was based on Hungarian method. After formulating the non convex EE maximization problem by utilizing the 
combination of parametric transformation, Lagrange duality and golden section search method, the sensing time and power allocation are obtained at each iteration in IDS algorithm. Simulations results shows the performance of our proposed scheme in comparison with some others existing scheme. However, the malicious users effects in CRN is not taken in consideration in this paper, which would be our future investigation in order to ensure better performance with secure communication.

\section{References}

[1] Letaief KB, Zhang W. Cooperative communications for cognitive radio networks. Proc IEEE 2009;97(5)):878-93.

[2] A. Ghasemi and E. S. Sousa, Collaborative spectrum sensing for opportunistic access in fading environments, In: Proceedings of 2005 first IEEE interna- tional symposium new frontiers in dynamic spectrum access networks, 2005. DySPAN 2005., p. 131-136, 2005.

[3] G. Ganesan. and Y. Li, Cooperative spectrum sensing in cognitive radio networks, In: Proceedings of 2005 first IEEE international symposium new frontiers in dynamic spectrum access networks, 2005. DySPAN 2005, p. 137-143, 2005.

[4] Ma J, Zhao G, Li Y. Soft Combination and Detection for cooperative spectrum sensing in cognitive radio networks. IEEE Trans Wirel Commun 2008;7(11): 4502-4507.

[5] Quan Z, Cui S, Sayed AH. Optimal Linear Cooperation for spectrum sensing in cognitive radio networks. Sel Top Signal Process IEEE J 2008;2(1):28-40.

[6] Liang Y-C, Zeng Y, Peh EC, Hoang AT. Sensing-throughput tradeofffor cognitive radio networks. IEEE Trans Wirel Commun 2008;7(4):1326-37.

[7] Moghimi F, Mallik RK, Schober R. Sensing Time and power optimization in MIMO cognitive radio networks. IEEE Trans Wirel Commun 2012;11(9):3398-408.

[8] Wu X, Xu J-L, Chen M, Wang J. Optimal Energy-efficient sensing and power allocation in cognitive radio networks. Math Probl Eng 2014;2014 Hindawi Publishing Corporation. 378 D. Das, S. Das/ Computers and Electrical Engineering 52 (2016) 362-378

[9] Almalfouh SM, Stuber GL. Interference-aware power allocation in cognitive radio networks with imperfect spectrum sensing. IEEE Trans Veh Technol 2011;60(4):1699713.

[10] Chatterjee S, Maity SP, Acharya T. Energy efficient cognitive radio system for joint spectrum sensing and data transmission. IEEE J Emerg Sel Topics Circuits Syst Sept. 2014;4(3):292300 .

[11] Naeem M, Illanko K, Karmokar A, Anpalagan A, Jaseemuddin M. Optimal power allocation for green cognitive radio: fractional programming approach. IET Commun 2013;7(12):1279-86.
[12] Naeem M, Illanko K, Karmokar A, Anpalagan A, Jaseemuddin M. Decode and forward relaying for energy-efficient multiuser cooperative cognitive radio network with outage constraints. IET Commun 2014;8(5):578-86.

[13] Haykin S. Cognitive radio: brain-empowered. Sel Areas Commun, IEEE J 2005; 23(2):201-20.

[14] Akyildiz IF, Lee W-Y, Vuran MC, Mohanty S. NeXt generation/dynamic spectrum access/cognitive radio wireless networks: a survey. Comput Netw 2006;50(13):2127-59.

[15] Kang X, Zhang R, Liang Y-C, Garg HK. Optimal power allocation strategies for fading cognitive radio channels with primary user outage constraint. IEEE J Sel Areas Commun 2011;29(2):374-83.

[16] Li X, Cao J, Ji Q, Hei Y. Energy Efficient Techniques with sensing time optimization in cognitive radio networks. Wirel Commun Netw Conf (WCNC 2013;2013:25-8.

[17] Yoon S-U, Ekici E. Voluntary spectrum handoff: a novel approach to spectrum management in CRNs. In: Proceedings of 2010 IEEE international con- ference communications (ICC); 2010. p. 1-5. [18] Luenberger DG, Ye Y. Linear and nonlinear programming. Springer Science \& Business Media; 2008.

[18] Ghasemi A, Sousa ES. Asymptotic performance of collaborative spectrum sensing under correlated log-normal shadowing. Commun Lett, IEEE 2007;11(1):34-6.

[19] Ren D, Ge J, Li J. Secondary user selection scheme using adaptive genetic algorithms for cooperative spectrum sensing under correlated shadowing. Wirel Pers Commun 2013;71(1):769-88. [21] Mai DTT, Chung TC, Nguyen D-T. Improving cooperative spectrum sensing under correlated lognormal shadowing. In: Proceedings of 2010 interna- tional conference cyber-enabled distributed computing and knowledge discovery (CyberC); 2010. p. 365-70.

[20] Gudmundson M. Correlation model for shadow fading in mobile radio systems. Electron Lett 1991;27(23):2145-6.

[21] Kuhn HW. The hungarian method for the assignment problem. Naval Res Logist Q 1955;2(1-2):83-97.

[22] Dinkelbach W. On nonlinear fractional programming. Manag Sci 1967;13(7):492-8. [25] Boyd S, Vandenberghe L. Convex optimization. Cambridge University Press; 2004.

[23] Mohammed El-Absi, Ali Ali, Mohamed El-Hadidy, and Thomas Kaiser Energy-Efficient Resource Allocation Based onInterference Alignment in MIMO-OFDM Cognitive Radio Networks. CROWNCOM 2015, LNICST 156, pp. 534-546, 2015.

[24] Deepa Das, Susmita Das. Optimal resource allocation for soft decision fusion-based cooperative spectrum sensing in cognitive radio networks Computers and Electrical Engineering 52 (2016) 362-378.

[25] Mohadeseh Soleimanpour-moghadam, Mohammad Askarizadeh, Siamak Talebi, and Shima Esmaeili. (2018). Low Complexity Green Cooperative Cognitive Radio Network With Superior Performance. IEEE Systems Journal. PP. 1-12. 10. 1109/JSYST. 2018. 2825281. 